\title{
Low-dose dacarbazine-doxorubicin therapy against intra-abdominal desmoid tumors
}

\author{
HIROFUMI YAMAMOTO $^{1}{ }^{\text {, RYOTA OSHIRO }}{ }^{1}$, JUNICHI NISHIMURA ${ }^{1}$, MAMORU UEMURA ${ }^{1}$, \\ NAOTSUGU HARAGUCHI ${ }^{1}$, TAISHI HATA ${ }^{1}$, ICHIRO TAKEMASA ${ }^{1}$, TSUNEKAZU MIZUSHIMA ${ }^{1}$, \\ MITSUGU SEKIMOTO ${ }^{2}$, YUICHIRO DOKI ${ }^{1}$ and MASAKI MORI ${ }^{1}$ \\ ${ }^{1}$ Department of Surgery, Gastroenterological Surgery, Graduate School of Medicine, Osaka University, Osaka; \\ ${ }^{2}$ National Hospital Organization, Osaka National Hospital, Osaka, Japan
}

Received September 19, 2012; Accepted December 3, 2012

DOI: $10.3892 /$ or.2013.2345

\begin{abstract}
Intra-abdominal desmoid tumor is a lifethreatening disease. Studies have shown that dacarbazine (DTIC)-doxorubicin (DOX) (D-D) therapy is the most effective treatment. However, myelosuppression is a major problem, and cardiac muscle disorders due to DOX limit the number of administration cycles, whereas it usually requires a long time to achieve tumor shrinkage. To resolve these issues, we introduced low-dose D-D therapy to 3 patients employing $50 \mathrm{mg} / \mathrm{m}^{2}$ DOX and $600-700 \mathrm{mg} / \mathrm{m}^{2}$ DTIC per cycle, which permits repeated administration cycles up to 10-11 times. Case 1 was a 23-year-old female with a sporadic recurrent mesenterium desmoid tumor located in the pelvis (maximum diameter, $8 \mathrm{~cm}$ ). Cases 2 and 3 were a 33-year-old female and a 36-year-old male. Both patients had intra-abdominal mesenterium desmoid tumors (maximum diameter 9.6 and $9.0 \mathrm{~cm}$, respectively) that were generated after proctocolectomy due to familial adenomatous polyposis. No severe adverse events occurred during the therapy. With the aid of sulindac and tamoxifen after low-dose D-D therapy, the first two patients achieved a complete response, and the third patient achieved a partial response and awaits further tumor shrinkage. Our experience indicates that low-dose DT-D therapy is a safe and effective regimen for patients with intra-abdominal desmoid tumors.
\end{abstract}

Correspondence to: Dr Hirofumi Yamamoto, Department of Surgery, Gastroenterological Surgery, Graduate School of Medicine, Osaka University, 2-2 Yamada-oka, Suita-City, Osaka 565-0871, Japan

E-mail: hyamamaoto@gesurg.med.osaka-u.ac.jp

Abbreviations: CT, computed tomography; DOX, doxorubicin; DTIC, dacarbazine; D-D therapy, DTIC-DOX therapy; FAP, familial adenomatous polyposis; NSAID, non-steroidal anti-inflammatory drug

Key words: doxorubicin, dacarbazine, intra-abdominal desmoid, low-dose therapy

\section{Introduction}

Desmoids are benign fibromatoses, composed histologically of mature fibroblasts. The tumors usually grow slowly. For extraabdominal desmoids, local control may be mainly achieved by surgical intervention and may be improved with the addition of radiation therapy, considering functional and cosmetic outcomes $(1,2)$. However, intra-abdominal desmoid tumors are life-threatening. Although desmoids do not metastasize to other organs, they grow and invade locally and infiltrate into surrounding organs such as the intestine, ureters and major vessels, causing bowel and ureteric obstruction and intratumoral hemorrhage.

In patients with familial adenomatous polyposis (FAP), desmoid tumors develop approximately 1,000 times more frequently than in the general population, among whom they are rare (3). Desmoid tumors develop in 3.6-13\% of patients with FAP (4), and account for 10-14\% of deaths of FAP patients, making it the second leading cause of death after colorectal carcinoma $(3,5)$. Surgery is usually indicated for extraabdominal desmoids such as those in the abdominal wall, but it is not recommended for mesenteric desmoid tumors, which account for the majority of intra-abdominal desmoids, since they are difficult to curatively resect. Surgery carries a high risk of serious complications such as bleeding, injury of the involved organs and short bowel syndrome, and it is associated with a high incidence of recurrence $(6,7)$.

Dacarbazine (DTIC)-doxorubicin (DOX) therapy (D-D therapy) is a hopeful therapeutic option against desmoid tumors, providing a high clinical response (8-12). DOX interferes with the function of topoisomerase II and DTIC is an $\mathrm{N}$-methyl-type compound that produces alkylating species. It was demonstrated that adding DTIC to DOX to treat sarcoma patients resulted in a better clinical outcome than DOX alone (13). The adverse effects of this protocol are well documented (14). Myelosuppression occurs as one of the major problems of this therapy. In addition, the risk of heart failure due to DOX limits the number of administration cycles although it usually takes a long time for patients with desmoid tumors to reach remission. Based on the original protocol proposed by Patel et al (10), DTIC (750-1,000 mg/m²/cycle) and DOX (60-90 mg/m²/cycle) are administered every 3-4 


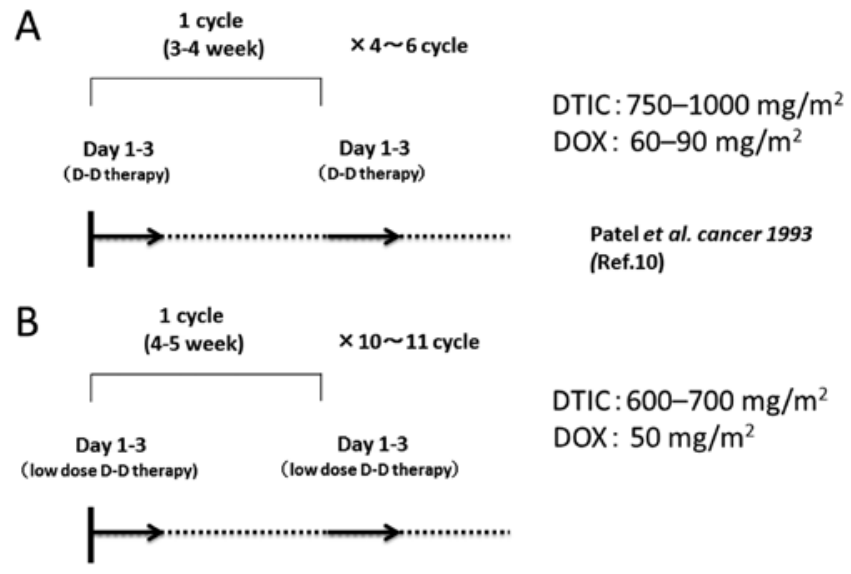

Figure 1. Scheme of dacarbazine-doxorubicin therapy. (A) Original D-D (dacarbazine-doxorubicin) combination therapy. (A) Low-dose D-D therapy. (DTIC, dacarbazine; DOX, doxorubicin. D-D, DTIC-DOX).

weeks for a total of 4-6 times (Fig. 1A), since cardiac muscle damage may occur if the DOX dose exceeds $550 \mathrm{mg} / \mathrm{m}^{2}$ (14).

To reduce the myelosuppression and assure an extended therapeutic period, we modified the protocol adopting lowdose DTIC (600-700 mg/m²/cycle) and DOX $\left(50 \mathrm{mg} / \mathrm{m}^{2} /\right.$ cycle $)$. We aimed to accomplish 10-11 therapeutic cycles. In this study, we report the successful treatment of 3 patients with intra-abdominal desmoids by the modified regimen that was safe and effective against desmoid tumors.

\section{Case reports}

General treatment and results. Low-dose DTIC-DOX (D-D) therapy was approved by the Institutional Review Board for Patients, and all expenses for DTIC and DOX therapy were paid by the Medical School Experience of the Osaka University Hospital. Low-dose D-D therapy was administered to 3 patients with intra-abdominal desmoid tumors: one sporadic and two FAP-associated tumors. For each cycle, DTIC and DOX were administered by continuous infusion by the central vein route for 3 days $(72 \mathrm{~h}$ ), repeated every 4-5 weeks (Fig. 1B). A total of 10-11 cycles of low-dose D-D therapy was administered. Two patients whose followup period after therapy was more than 4 years achieved a complete response (CR). The third patient whose last administer rate was completed in September 2011 had a partial response (PR). To monitor for heart dysfunction caused by DOX, electric cardiography and echocardiogram were repeated periodically, but no adverse effects were detected. Other adverse events were only slight ones: nausea, vomiting, alopecia and leukocytopenia without fever. All patients were able to spent $\sim 20$ days at home in a month during low-dose D-D therapy.

\section{Individual treatments}

Case 1. In 1997, a 23-year-old female was found to have a $5.5-\mathrm{cm}$ size sporadic desmoid tumor in the pelvic space and she underwent surgery. Three years later the recurrent tumor appeared in her pelvis, and she underwent a second surgery. In September 2005, she again had recurrent disease, and the

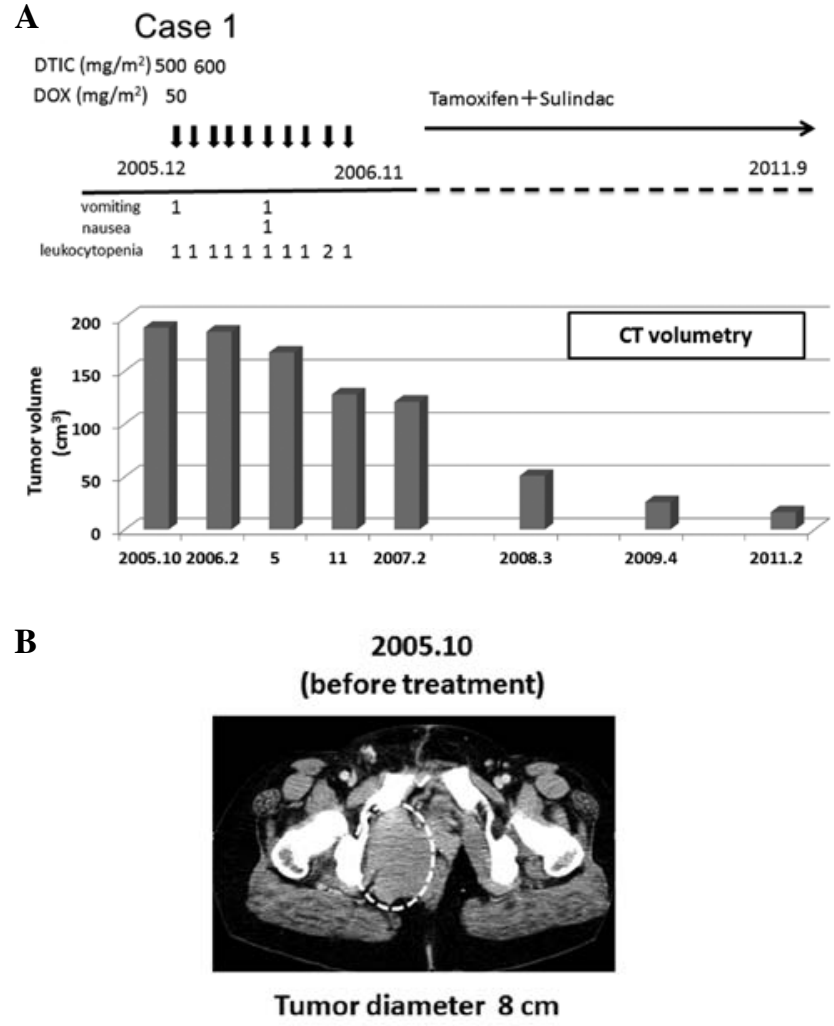

2007.2 (after 10 cycles)

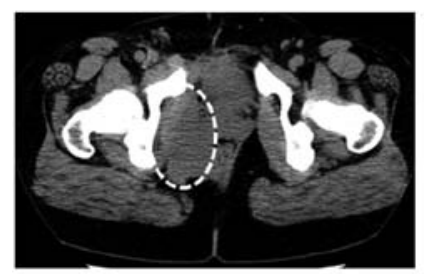

Tumor diameter $5.4 \mathrm{~cm}$

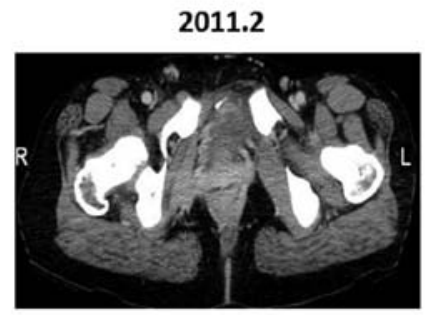

Remnant scar
Figure 2. Summary of case 1. (A) Regimen, adverse events and tumor volume measured by CT volumetry are shown in a time-dependent manner. (B) CT scan images. Circle indicates desmoid tumor in the pelvis. The desmoid tumor compressed the rectum to the left side.

tumor was no longer resectable due to invasion to the vagina and rectum. Her principal doctor consulted our team. In October 2005, the computed tomography (CT) scan image indicated that the desmoid tumor (maximum diameter, $8 \mathrm{~cm}$ ) occupied the right half of the lower pelvis and it compressed the rectum far to the left side (Fig. 2B). From December 2005 to November 2006, low-dose D-D therapy was performed every $4-5$ weeks and $500-600 \mathrm{mg} / \mathrm{m}^{2}$ DTIC and $50 \mathrm{mg} / \mathrm{m}^{2}$ DOX was administered 10 times (Fig. 2A). According to the Common Terminology Criteria for Adverse Events (CTCAE) v4.0 by the National Cancer Institute (15), the patient experienced grade 1 vomiting and grade 1 nausea (Fig. 2A). The patient had grade 1-2 leukocytopenia (Fig. 2A, CTCAE v3.0) (15) but had no febrile neutropenia (CTCAE v4.0). An anti-estrogen receptor (tamoxifen, 40-20 mg/day) and the NSAID sulindac (Clinoril, $300 \mathrm{mg} /$ day) were subsequently administered orally. After 10 cycles, the tumor diameter was 
A

Case 2

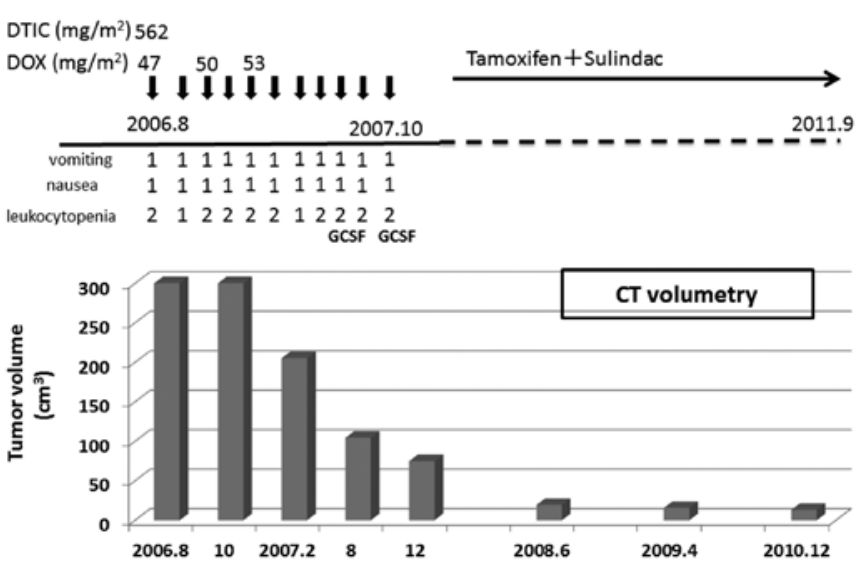

B

2006.8

(before treatment)

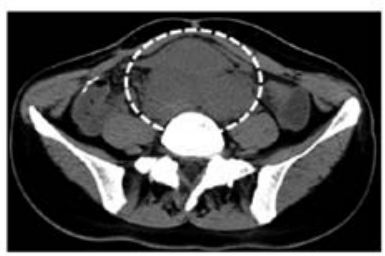

Tumor diameter $9.6 \mathrm{~cm}$

2007.12

(after 11 cycles)

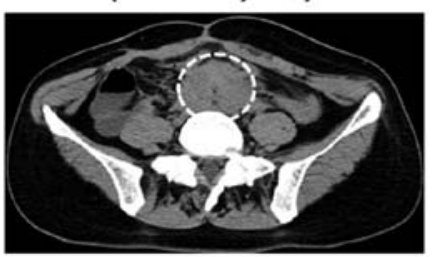

Tumor diameter $5.5 \mathrm{~cm}$

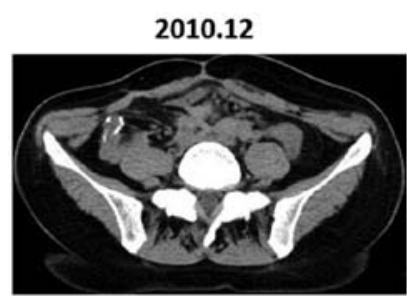

Remnant scar

Figure 3. Summary of case 2. (A) Regimen, adverse events and tumor volume measured by CT volumetry are shown in a time-dependent manner. (B) CT scan images. Circle indicates desmoid tumor.

reduced to $5.4 \mathrm{~cm}$, and the tumor volume decreased by $36.6 \%$ when analyzed by CT volumetry (Fig. 2B). In February 2011, the desmoid tumor almost disappeared, and the rectum was located back to the middle site (Fig. 2B). The patient has not had a recurrent tumor up until August 2012.

Case 2. In November 2004, a 33-year-old female underwent proctocolectomy due to FAP. In August 2006, an intraabdominal desmoid tumor $(9.6 \mathrm{~cm}$ in diameter) derived from the mesenterium was found (Fig. 3A). From August 2006 to October 2007, low-dose D-D therapy $\left(562.5 \mathrm{mg} / \mathrm{m}^{2}\right.$ DTIC and $47.0-53.0 \mathrm{mg} / \mathrm{m}^{2}$ DOX per cycle) was executed for a total of 11 cycles, followed by oral administration of an anti-estrogen receptor (tamoxifen, 40-20 mg/day), and the NSAID sulindac (Clinoril,300 mg/day) (Fig. 3A). During the therapeutic period, the patient experienced grade 1 nausea and grade 1 vomiting (CTCAE v4.0, Fig. 3A). She had no febrile neutropenia. Instead, the patient had grade 1 or 2 leukocytosis (CTCAE

A

\section{Case 3}

DTIC $\left(\mathrm{mg} / \mathrm{m}^{2}\right) \quad 700$

$\operatorname{DOX}\left(\mathrm{mg} / \mathrm{m}^{2}\right) \quad 50$

50 Sulindac

$\downarrow \downarrow \downarrow \downarrow \downarrow \downarrow \downarrow \downarrow \downarrow$ 2011.

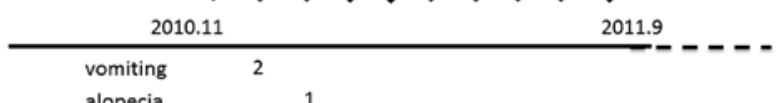

alopecia 1

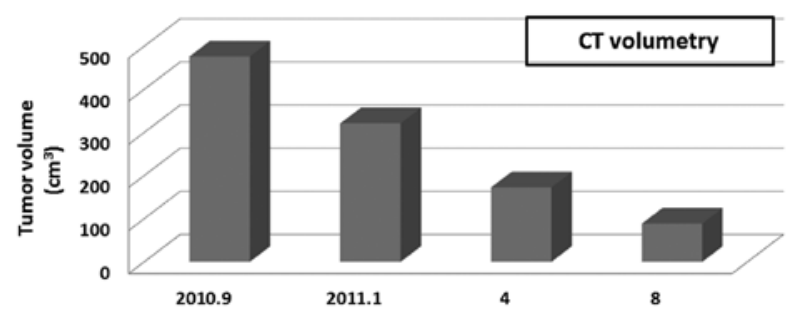

B

2010.9

(before treatment)

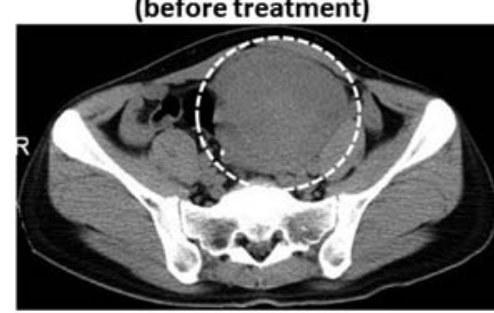

Tumor diameter $9 \mathrm{~cm}$

2011.8

(after 9 cycles)

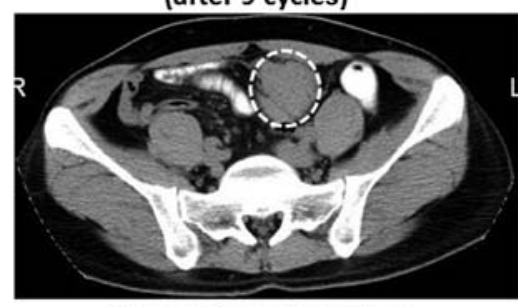

Tumor diameter $5.3 \mathrm{~cm}$

Figure 4. Summary of case 3. (A) Regimen, adverse events and tumor volume measured by CT volumetry are shown in a time-dependent manner. (B) CT scan images. Circle indicates desmoid tumor.

v3.0) which was alleviated promptly by treatment with granulocyte stimulating factor (GCSF) twice (Fig. 3A). In December 2007, the tumor volume was decreased by $83.75 \%$ (Fig. 3B). The desmoid tumor continued to shrink and in December 2010, CT scan imaging showed only a remnant scar (Fig. 3B). The patient has not had a recurrent tumor up until June 2012 .

Case 3. In February 2006, a 33-year-old male underwent proctocolectomy due to FAP. In September 2010, an intraabdominal desmoid tumor $(9.0 \mathrm{~cm}$ in diameter) generated from the mesenterium was found by CT scan (Fig. 4B). From November 2010 to September 2011, low-dose D-D therapy $\left(700 \mathrm{mg} / \mathrm{m}^{2}\right.$ DTIC and $50 \mathrm{mg} / \mathrm{m}^{2}$ DOX) was administered 10 times, followed by oral administration of the NSAID sulindac (Clinoril, $300 \mathrm{mg} /$ day) (Fig. 4A). During the therapeutic period, blood toxicity was not experienced. The patient experienced grade 2 vomiting and grade 1 alopecia (CTCAE v4.0) (Fig. 4A). In August 2011, the tumor volume decreased by $81.4 \%$ (Fig. 4 B). 


\section{Discussion}

Dacarbazine-doxorubicin (DTIC-DOX) therapy achieved better results against desmoid tumors (8-12) than other potential treatments such as prednisolone, interferon $\gamma$, vinorelbine, vinblastine and methotrexate (16-20). We previously reported the case of a patient who repeatedly had aggressive intraabdominal mesenteric desmoids after proctocolectomy due to FAP. He was administered $667-800 \mathrm{mg} / \mathrm{m}^{2}$ DTIC and $67-80 \mathrm{mg} / \mathrm{m}^{2}$ DOX in each cycle. Although this DTIC-DOX therapy in a total of 7 courses achieved tumor inhibitory effects, the patient experienced grade 3 to 4 leukocytopenia (CTCAE v3.0) 5 times in 7 therapies, and at the time GCSF was necessary. Moreover, during the tumor regression, the patient had a fever of higher than $38^{\circ} \mathrm{C}$, which persisted for 2 months.

Apart from severe leukocytopenia, one adverse effect of DTIC-DOX therapy is heart dysfunction due to DOX. Following the original regimen by Patel et al (10) (DTIC, $750-1000 \mathrm{mg} / \mathrm{m}^{2}$; DOX, $60-90 \mathrm{mg} / \mathrm{m}^{2}$ ), drug administration is limited to 4-6 cycles for fear of heart failure. Paradoxically, it usually takes an extended time for the desmoid tumor to reach complete remission. Therefore, we planned to administer a reduced dose of DTIC and DOX at $600-700$ and $50 \mathrm{mg} / \mathrm{m}^{2}$, respectively, per cycle. According to this protocol, we were able to continue therapy every 4-5 weeks for 10-13 months (10-11 cycles).

The first two patients after a long follow-up period achieved a complete response (CR). Case 2 patient had no febrile neutropenia and had an absolute neutrophil count $<1000$ without fever, which was treated with GCSF twice. Case 2 patient also did not have febrile neutropenia but did not require GCSF throughout the therapeutic period. Both patients spent 1 week in the hospital and 3-4 weeks at home during each cycle period.

According to RECIST guidelines, a 30\% reduction in tumor diameter $(2 r)$ for partial response is equivalent to $65 \%$ reduction in tumor volume $\left(4 / 3 \pi r^{3}\right)(21)$. However, the desmoid tumor of case 1 patient had only a $12.2 \%$ reduction in volume 4 months after commencement of the therapy, and the desmoid tumor of case 2 patient had a $14.3 \%$ reduction in volume after 5 months (Figs. 2A and 3A). Many physicians may discontinue therapy at this point as these patients did not achieve a partial response even after 4-5 months from the start of the treatment. However, considering our previous experience of patients treated with a standard dose of D-D therapy, the desmoid tumors of the patients did not respond to therapy until 4 months and subsequently continued to shrink for a long time. Therefore, we continued low-dose D-D therapy by monitoring the tumor volume carefully and their desmoid tumors continued to shrink, eventually achieving almost a CR. Consequently, case 1 patient received 10 cycles for 12 months, and case 2 patient received 11 cycles of low-dose D-D therapy for 14 months. CR was maintained for 1.5 and 2.5 years, respectively.

After D-D therapy, we administered NSAID sulindac and an anti-estrogen receptor tamoxifen to facilitate tumor shrinkage. These agents are reportedly shown to be effective in $15-30 \%$ of desmoid tumor cases $(22,23)$. Since the desmoid tumor of case 1 patient markedly shrank after low-dose D-D therapy with the aid of sulindac and tamoxifen, we cannot deny the possibility that additional administration of these supplementary agents might also be of importance for treating desmoid tumors.

We are currently treating the third patient who had intraabdominal desmoid after proctocolectomy due to FAP with low-dose D-D therapy (Fig. 4A). The tumor volume of this patient decreased by $32.6 \%$ at 2 months and by $81.4 \%$ at 9 months following commencement of low-dose D-D therapy, without any serious adverse events (Fig. 4).

Taken together, we showed that low-dose DTIC-DOX therapy is safe and effective and ensures an acceptable quality of life to the patients who need to endure a long therapy period. Further large scale confirmation study is essential.

\section{References}

1. de Bree E, Keus R, Melissas J, Tsiftsis D and van Coevorden F: Desmoid tumors: need for an individualized approach. Expert Rev Anticancer Ther 9: 525-535, 2009.

2. Berri RN, Baumann DP, Madewell JE, Lazar A and Pollock RE: Desmoid tumor: current multidisciplinary approaches. Ann Plast Surg 67: 551-564, 2011.

3. Gurbuz AK, Giardiello FM, Petersen GM, Krush AJ, Offerhaus GJ, Booker SV, et al: Desmoid tumors in familial adenomatous polyposis. Gut 35: 377-381, 1994.

4. Clark SK and Phillips RK: Desmoids in familial adenomatous polyposis. Br J Surg 83: 1494-1504, 1996.

5. Arvanitis ML, Jagelman DG, Fazio VW, Lavery IC and McGannan E: Mortality in patients with familial adenomatous polyposis. Dis Colon Rectum 33: 639-642, 1990.

6. Clark SK, Neale KF, Landgrebe JC and Phillips RK: Desmoid tumours complicating familial adenomatous polyposis. Br J Surg 86: 1185-1189, 1999.

7. Heiskanen I and Jarvinen HJ: Occurrence of desmoid tumours in familial adenomatous polyposis and results of treatment. Int $\mathbf{J}$ Colorectal Dis 11: 157-162, 1996.

8. Gega M, Yanagi H, Yoshikawa R, Noda M, Ikeuchi H, Tsukamoto K, et al: Successful chemotherapeutic modality of doxorubicin plus dacarbazine for the treatment of desmoid tumors in association with familial adenomatous polyposis. J Clin Oncol 24: 102-105, 2006.

9. Lynch HT, Fitzgibbons R Jr, Chong S, Cavalieri J, Lynch J, Wallace F, et al: Use of doxorubicin and dacarbazine for the management of unresectable intra-abdominal desmoid tumors in Gardner's syndrome. Dis Colon Rectum 37: 260-267, 1994.

10. Patel SR, Evans HL and Benjamin RS: Combination chemotherapy in adult desmoid tumors. Cancer 72: 3244-3247, 1993.

11. Poritz LS, Blackstein M, Berk T, Gallinger S, McLeod RS and Cohen Z: Extended follow-up of patients treated with cytotoxic chemotherapy for intra-abdominal desmoid tumors. Dis Colon Rectum 44: 1268-1273, 2001.

12. Ezumi K, Yamamoto H, Takemasa I, Nomura M, Ikeda M, Sekimoto M and Monden M: Dacarbazine-doxorubicin therapy ameliorated an extremely aggressive mesenteric desmoid tumor associated with familial adenomatous polyposis: report of a case. Jpn J Clin Oncol 38: 222-226, 2008.

13. Gottlieb JA, Benjamin RS, Baker LH, O'Bryan RM, Sinkovics JG, Hoogstraten B, et al: Role of DTIC (NSC-45388) in the chemotherapy of sarcoma. Cancer Treat Rep 60: 199-203, 1976.

14. Gottieb JA, Baker LH, Quagliana JM, Luce JK, Whitecar JP Jr, Sinkovics JG, et al: Chemotherapy of sarcoma with a combination of adriamycin and dimethyl triazeno imidazole carboxamide. Cancer 30: 1632-1638, 1972.

15. CTCAE v3.0 and v4.0 provided by the National Cancer Institute. http://ctep.cancer.gov/protocolDevelopment/electronic_applications/ctc.htm\#ctc_40.

16. Nakada I, Ubukata H, Goto Y, Watanabe Y, Sato S, Tabuchi T, et al: Prednisolone therapy for intra-abdominal desmoid tumors in a patient with familial adenomatous polyposis. J Gastroenterol 32: 255-259, 1997. 
17. Bauernhofer T, Stoger H, Schmid M, Smola M and GurtlLackner B, Hofler G, et al: Sequential treatment of recurrent mesenteric desmoid tumor. Cancer 77: 1061-1065, 1996.

18. Kono T, Tomita I, Chisato N, Matsuda M, Kakisaka A and Kasai S: Successful low-dose chemotherapy using vinblastine and methotrexate for the treatment of an ileoanal pouch mesenteric desmoid tumor: report of case. Dis Colon Rectum 47: 246-249, 2004

19. Weiss AJ, Horowiz S and Lackman RD: Therapy of desmoid tumors and fibromatosis using vinorelbine. Am J Clin Oncol 22 193-195, 1999.

20. Okuno SH and Edmonson JH: Combination chemotherapy for desmoid tumors. Cancer 97: 134-135, 2003.
21. Therasse P, Arbuck SG, Eisenhauer EA, Wanders J, Kaplan RS, Rubinstein L, Verweij J, Glabbeke MV, van Oosterom AT, Christian MC and Gwyther SG: New guidelines to evaluate the response to treatment in solid tumors. European Organization for Research and Treatment of Cancer, National Cancer Institute of the United States, National Cancer Institute of Canada. J Natl Cancer Inst 92: 205-216, 2000.

22. Hansmann A, Adolph C, Vogel T, Unger A and Moeslein G: High-dose tamoxifen and sulindac as first-line treatment for desmoid tumors. Cancer 100: 612-620, 2004.

23. Patel SR and Benjamin RS: Desmoid tumors respond to chemotherapy: defying the dogma in oncology. J Clin Oncol 24: 11-12, 2006. 